\title{
A limited spectrum of phenylalanine hydroxylase mutations is observed in phenylketonuria patients in western Poland and implications for treatment with $6 \mathrm{R}$ tetrahydrobiopterin
}

\begin{abstract}
Steven F Dobrowolski ${ }^{1}, \mathrm{~K}$ Borski $^{2}$, CC Ellingson ${ }^{3}, \mathrm{R} \mathrm{Koch}^{4}, \mathrm{HL}$ Levy $^{5}$ and EW Naylor ${ }^{6}$
Phenylketonuria (PKU) is an autosomal recessive defect in hepatic metabolism of phenylalanine, which is secondary to mutations in the phenylalanine hydroxylase (PAH) gene. Sixty-seven ethnically Polish PKU patients, followed at the Outpatient Department of Pediatrics and Developmental Medicine in Poznan, Poland, were assessed for mutations in the PAH gene. Two mutations were identified in 61 of 67 patients and a single mutation was identified in the remaining six patients. The four most prevalent mutations (p.R408W, 68\%; c.1066-11G >A, 6\%; c.1315+1G >A, 5.2\%; c.822-832delGCCCATGTATA, 3.7\%) accounted for $83 \%$ of the mutant alleles. Fifteen additional mutations were identified of which most (13/15) were observed in an individual patient. Before knowledge of $P A H$ genotypes, 19 patients were challenged with a $20 \mathrm{mg} \mathrm{kg}^{-1}$ dose of $6 \mathrm{R}$ tetrahydrobiopterin $\left(\mathrm{BH}_{4}\right)$ and serum phenylalanine concentration was monitored in hospital over $24 \mathrm{~h}$. Two patients responded to the $\mathrm{BH}_{4}$ challenge with a reduction of serum phenylalanine concentration $>30 \%$ from baseline. $P A H$ genotypes of the two responsive patients uld have been predicted, as they contained mutations recognized as $\mathrm{BH}_{4}$ responsive, whereas the 17 patients who were unresponsive would have been predicted as their mutations were either recognized as non-responsive or were highly deleterious frame-shift mutations. Overall, only $7.5 \%(5 / 67)$ of patients had $P A H$ mutations recognized as responsive to co-factor therapy. Among the PKU patients from western Poland, $\mathrm{PAH}$ mutations responsive to $\mathrm{BH}_{4}$ therapy are poorly represented; therefore, genotyping may be useful for identifying candidate patients likely to respond to $\mathrm{BH}_{4}$ before physiological challenge.
\end{abstract}

Journal of Human Genetics (2009) 54, 335-339; doi:10.1038/jhg.2009.37; published online 15 May 2009

Keywords: $\mathrm{BH}_{4}$ response; genotyping; melt profiling; $\mathrm{PAH}$; PKU

\section{INTRODUCTION}

Phenylketonuria (MIM261600, EC 1.14.16.1) results from defects in the liver enzyme, phenylalanine hydroxylase $(P A H)$. Newborn screening uses blood dried on filter paper (dried blood spot, DBS) to prospectively identify PKU-affected newborns, through an increased concentration of phenylalanine. ${ }^{1,2}$ An increased phenylalanine concentration identifies a spectrum of diseases ranging from mild hyperphenylalanemia, in which blood phenylalanine concentration is $180-600 \mathrm{~m} \mathrm{l}^{-1}$ to classical PKU in which the phenylalanine concentration is greater than $1200 \mu \mathrm{Ml}^{-1}$. The treatment goal for newborns and children is maintaining serum phenylalanine concentration between 120 and $360 \mu \mathrm{M} \mathrm{I}^{-1}$, which has traditionally been achieved with a phenylalanine-restricted diet. $^{3}$ In a subset of PKU patients, phenylalanine concentration is manageable with pharmacological doses (typically $20 \mathrm{mg} \mathrm{kg}^{-1} \mathrm{day}^{-1}$ ) of the co-factor 6R-tetrahydrobiopterin $\left(\mathrm{BH}_{4}\right)$ with either limited or no dietary restriction. ${ }^{4-7}$ Life-long patient compliance with traditional dietary restriction is poor as upward of $75 \%$ of adolescent and adult patients do not comply with the dietary restriction, leading to neurological damage from uncontrolled phenylalanine concentrations. ${ }^{8}$ For patients whose disease responds to $\mathrm{BH}_{4}$, co-factor therapy is an attractive means to increase patient compliance.

$P A H$ genotyping has the utility to diagnose PKU and to some extent the genotype has value in disease categorization (classical PKU, mild PKU, mild hyperphenylalanemia, etc) and predicting the potential for response to co-factor therapy. ${ }^{9-14} \mathrm{PAH}$ mutations are spread throughout the gene and the spectrum of mutations varies among PKU patients from different geographical regions. For example, in the heterogeneous American population the spectrum of mutations is exceedingly broad with p.R408W present at $\sim 18 \%$ and a small number of other mutations are observed at $6-8 \%{ }^{12}$ In other geographic regions, the distribution of mutations is more

${ }^{1}$ Department of Pathology, University of Utah School of Medicine, Salt Lake City, UT, USA; ${ }^{2}$ Poznan University of Medical Sciences, Poznan, Poland; ${ }^{3}$ Penn State Hershey Medical Center, Hershey, PA, USA; 'Women and Children's Hospital, University of Southern California, Los Angeles, CA, USA; ${ }^{5}$ Children's Hospital of Boston, Boston, MA, USA and ${ }^{6}$ NGS Research \& Development-Europe, GmbH, Westhafenplatz 1, Frankfurtam Main, Germany 
restricted. ${ }^{15-18}$ If a population presented with a high prevalence of $\mathrm{PAH}$ mutations unresponsive to co-factor therapy, this would limit the utility of $\mathrm{BH}_{4}$ for treating $\mathrm{PKU}$ within that population.

$P A H$ genotypes were determined for 67 ethnically Polish PKU patients, followed at the PKU Clinic in the Outpatient Department of Pediatrics and Developmental Medicine, Poznan, Poland. Mutations recognized as responsive to co-factor therapy were observed in a minority of patients (5/67) and the vast majority of mutations were recognized as $\mathrm{BH}_{4}$ non-responsive or novel frame-shift mutations that could be assumed to also be non-responsive. A single dose, $\mathrm{BH}_{4}$ challenge $\left(20 \mathrm{mg} \mathrm{kg}^{-1}\right)$ was administered to 19 patients before knowledge of the $\mathrm{PAH}$ genotypes. The genotypes of the two responsive patients ( $>30 \%$ reduction in phenylalanine concentration) would have predicted them as likely to respond. Mutations, among the 17 non-responsive patients, were predominantly recognized as non- $\mathrm{BH}_{4}$ responsive alleles. Genotypes of these patients would have identified them as unlikely to respond to co-factor therapy. PKU patients from western Poland may be well served by first determining the $P A H$ genotype to identify those that were more likely to respond to $\mathrm{BH}_{4}$ before physiological challenge.

\section{MATERIALS AND METHODS}

\section{Patients and collection of specimens}

Blood samples were collected on Guthrie filter paper cards from $67 \mathrm{PKU}$ patients (40 female and 27 male patients) followed at the PKU Clinic in Poznan, Poland. Patients were between 1 and 21 years of age and from 61 pedigrees. Appropriate IRB approval was obtained for all participants.

\section{Preparation of DNA from DBS}

DNA was prepared from DBS using a manual adaptation of the automated procedure described earlier. ${ }^{19}$ Quantification of the nucleic acids was not required as $1 \mu \mathrm{l}$ of DNA extract reliably supports PCR using the PAH panel. ${ }^{12}$

\section{Analysis of the PAH gene}

Analysis of the $P A H$ gene used a modification of the high-resolution melt profiling assay described earlier. ${ }^{12}$ Briefly, the 13 coding regions of the $P A H$ gene along with adjacent intron regions critical to mRNA processing are each amplified in an individual product in the presence of LCGreen Plus (Idaho Technology, Salt Lake City, UT, USA), a saturating dsDNA binding dye. Melt profiling was performed with a 96-well formatted LightScanner (Idaho Technology) using a melting range of $70-95^{\circ} \mathrm{C}$. Data are normalized, temperature corrected and converted to subtractive difference plots as described. ${ }^{12,20}$ Specimens whose melting profiles deviated from the wildtype control profiles are recovered from the plate and assessed by DNA sequencing. To expedite DNA sequencing, M13 DNA sequence tails were added to the $5^{\prime}$ end of the primer sets described earlier. ${ }^{12}$ The following primers were used to direct forward-strand and reverse-strand DNA sequencing respectively: 5'-GTAAAACGACGGCCAGT-3'; 5'-CAGGAAACAGCTAT GAC-3'. DNA sequence data were analyzed with Mutation Surveyor software (Soft Genetics, State College, Pennsylvania, PA, USA).

\section{Challenge with $\mathrm{BH}_{4}$}

In patients 1-19, a $24 \mathrm{~h} \mathrm{BH}_{4}$ loading test was performed using $20 \mathrm{mg} \mathrm{BH}_{4} \mathrm{~kg}^{-1}$ body mass. The loading tests were performed in the hospital. Phenylalanine concentrations in serum were measured before $\mathrm{BH} 4$ challenge $(T=0)$ and 4,8 and $24 \mathrm{~h}$ following the challenge.

\section{RESULTS}

\section{Melting profile assessment of the PAH gene}

Figure 1 provides representative melting profiles assessing $P A H$ exons 1, 7 and 12. The upper portion of each panel shows melting curves, whereas the lower portion displays the data as a subtractive difference plot. The subtractive difference plots are normalized to the wild-type control, such that other specimens with a wild-type gene cluster about the origin of the $y$ axis whereas profiles containing variants deviate from the origin and appear as peaks. Figure 1a assesses $P A H$ exon 1, identifying one deviating melt profile, which upon follow-up DNA sequencing was determined to possess the novel deletion c.47-48delCT. Several mutations are identified in Figure $1 \mathrm{~b}$ that assesses PAH exon 7. The novel mutation, c.822-832delGCC CATGTATA, is identified in the homozygous and heterozygous form and as a compound heterozygote with the $\mathrm{c} .735 \mathrm{G}>\mathrm{A}$ polymorphism (p.V245V). Figure 1c shows the assessment of the PAH exon 12, which harbored the largest percentage of mutations in the patients from western Poland. The heterozygous and homozygous p.R408W are readily discernable in Figure 1c.

Among the 67 patient samples, mutations were found within or adjacent to exons $1,3,4,5,7,11$ and 12; however, the majority of variants was observed in exons 7,11 and 12. Single mutations were found in exons 1, 3 and 5 (c.47-48delCT exon 1; p.R111X exon 3; p.Y166X, exon 5) whereas two variants were observed in the fragment assessing exon 4 (c.353-1G $>A$ and c.441+5G $>$ T). Variants were not observed in or adjacent to exons $2,6,8,9,10$ and 13 .

\section{Genotypes observed in 67 PKU patients}

Table 1 provides genotypes of the 67 ethnically Polish PKU patients and patients from the same pedigree displayed a common genotype. The p.R408W mutation was present in 60 of 67 patients. Twenty-nine patients were p.R408W homozygotes, 27 patients were compound heterozygotes p.R408W/other and four were p.R408W/unknown where a second variant was not identified. Two additional mutations described earlier were observed in multiple patients, c.1066-11G $>$ A was in compound heterozygous form with p.R408W in eight patients and c.1315+1G $>A$ was observed in seven patients (three patients p.R408W/c.1315+1G >A; one patient, c.1315+1G >A/p.P281L; one patient, c.1315+1G >A/p.Y166X; two patients, c.1315+1G >A/ unknown). The novel $11 \mathrm{bp}$ deletion c.822-832delGCCCATGTATA was observed in four patients from unrelated kindred. Patient no. 47 was homozygous for deletion, patients nos. 7 and 8 were compound heterozygotes for deletion and p.R408W, whereas patient no. 15 was a compound heterozygote for deletion and p.I306V. A single variant was identified in six patients (four patients, pR408W/unknown; two patients, c.1315+1G > A/unknown).

Table 2 shows the frequency of recurrent $P A H$ mutations in PKU patients from this study compared with recurrent mutations observed in PKU patients from neighboring regions. The p.R408W mutation is the most common in all regions, albeit at different frequencies. The c.822-832delGCCCATGTATA mutation was not identified outside western Poland. The p.R158Q mutation was common in the Czech Republic, Ukraine and Lithuania, whereas p.R261Q was common in eastern Germany and Slovakia, but neither was observed in the 67 patients from western Poland. ${ }^{15-18}$

\section{Response to $\mathrm{BH}_{4}$ challenge in patients 1-19}

Patients 1-19 were challenged with a $20 \mathrm{mg} \mathrm{kg}^{-1}$ oral dose of $\mathrm{BH}_{4}$ and serum phenylalanine concentration was determined at four time points ( $T=0,4,8$ and $24 \mathrm{~h}$ ) over a 24 -h period. Table 3 provides the genotypes, phenylalanine concentration at the time of $\mathrm{BH}_{4}$ challenge $(T=0)$, the percentage reduction from $T=0$ and the latency until maximum reduction of phenylalanine. Response to $\mathrm{BH}_{4}$ was defined as $a \geqslant 30 \%$ reduction in serum phenylalanine concentration from baseline $(T=0)$. Patient nos. 1 and 15 were responsive with 81 and $74 \%$ reductions in phenylalanine concentration respectively. Patient nos. 1 and 15 are compound heterozygotes each possessing one 
a
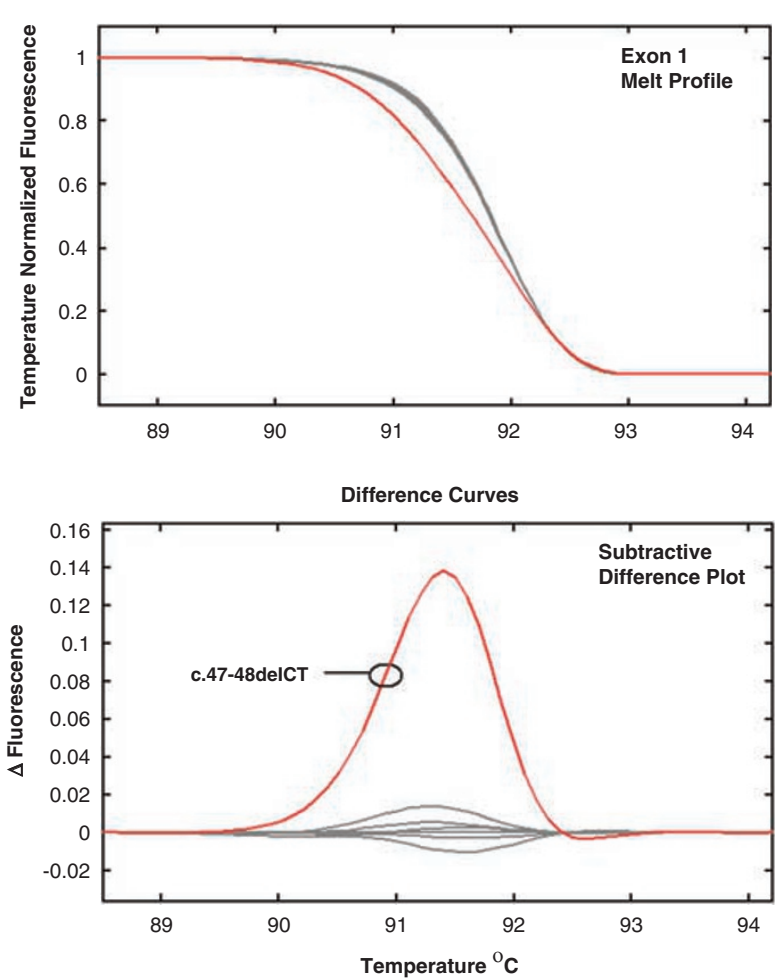

b Shifted Melting Curves
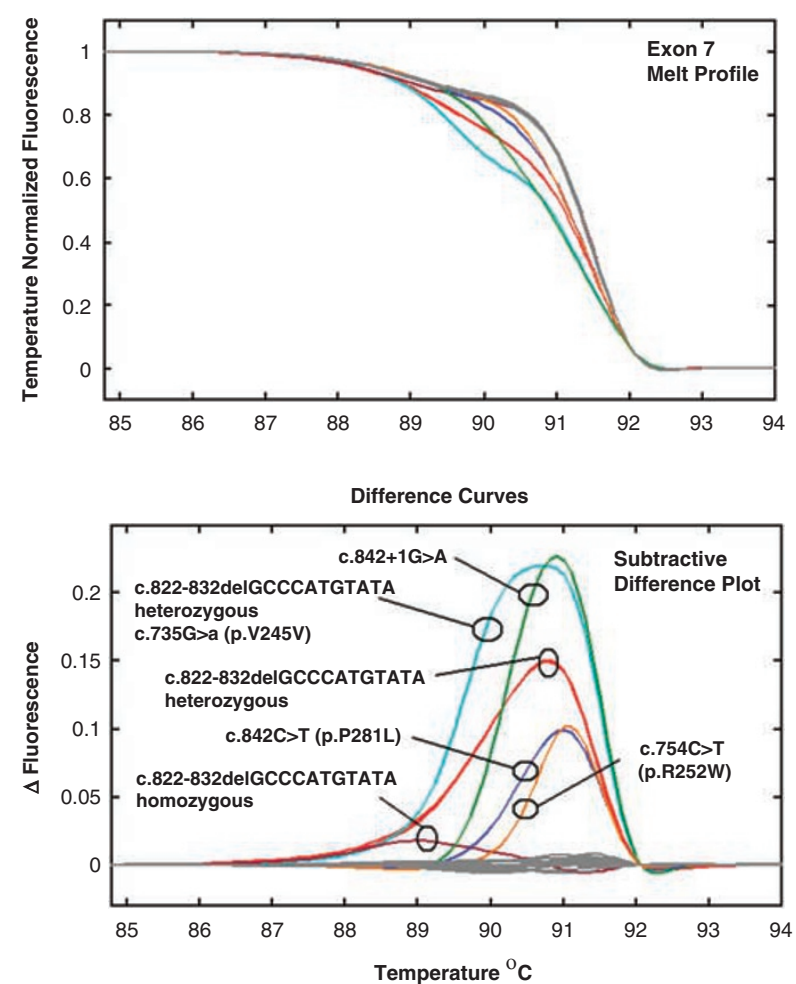

C
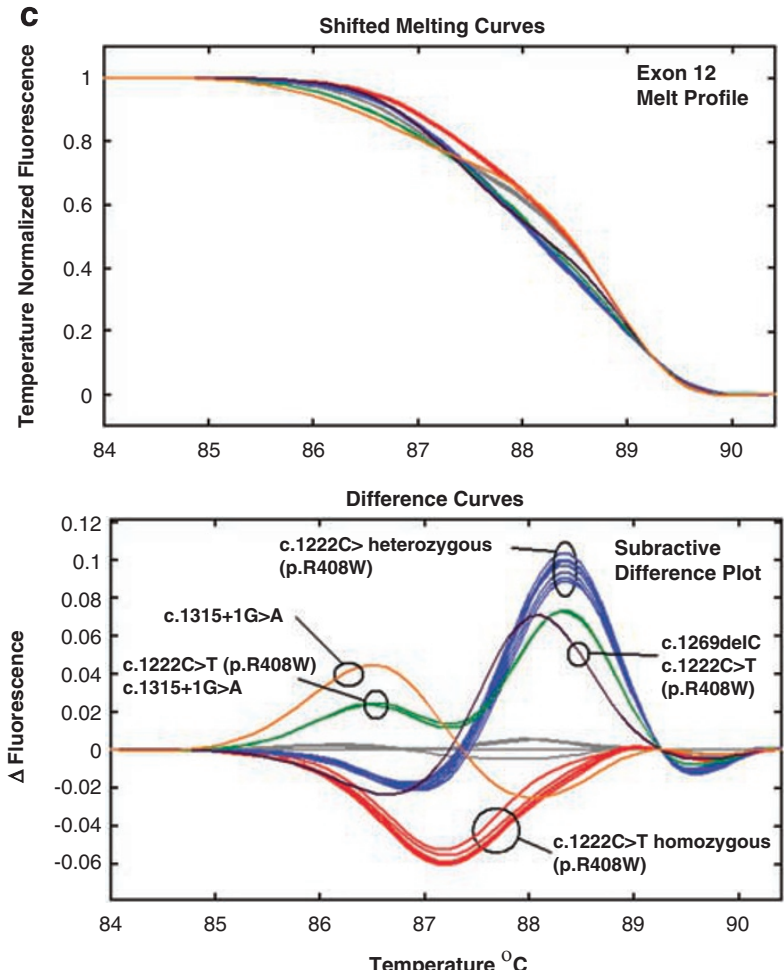

Figure 1 Melting profiles of $P A H$ exons 1,7 and 12. The upper panel is a melting curve whereas the lower panel provides the data analyzed as a subtractive difference plot. Subtractive difference plots are normalized to the wild-type (gray) profiles. (a) Exon 1. Gray, eight wild-type profiles; red. c.47-48delCT heterozygote. (b) Exon 7. Gray 12 profiles, wild type; purple, c.822-832delGCCCATGTATA homozygote; red, 2 profiles c.822-832delGCCCATGTATA heterozygote; blue, p.P281L heterozygote; orange, p.R252W heterozygote; green, c.842+1G>A heterozygote; pale blue c.822-832delGCCCATGTATA/ c.735G $>$ A compound heterozygous. (c) Exon 12. Gray 12 profiles, wild type; red 8 profiles, p.R408W homozygous; blue 10 profiles, p.R408W heterozygous; Orange c. $1315+1 \mathrm{G}>\mathrm{A}$ heterozygous; green, 2 profiles, c.1315+1G $>$ A/p.R408W compound heterozygous; purple c.1269delC/p.R408W compound heterozygous. 
mutation (p.E390G in patient no. 1 and p.I306V in patient no. 15) recognized as responsive to therapy with $\mathrm{BH}_{4} \cdot{ }^{5,13}$

\section{DISCUSSION}

PKU represents the paradigm for a treatable genetic disease. Diagnosis, classification and treatment of PKU has evolved over the past 40 years, and most significantly with the observation that a subset of patients may be treated with daily oral doses of $\mathrm{BH}_{4}{ }^{4-7}$ Assessing the $\mathrm{PAH}$ gene in candidate patients has utility in diagnosis, but also provides insight into disease classification and treatment. ${ }^{8-11}$ Mutations observed in PKU patients of differing ethnicity and geographic locations vary considerably. Overall, this study identified $95.5 \%$ of the mutant alleles in 67 ethnically Polish PKU patients, which is slightly lower than the $99 \%$ of mutant alleles identified by our group in 95 patients from the United States. ${ }^{12}$ Single exon $P A H$ deletions have been identified in PKU patients from Poland and the PCR-based assay used in this study would not identify deletions, especially so in heterozygous form. ${ }^{21}$ To

Table 1 Genotypes of PKU patients

\begin{tabular}{|c|c|}
\hline Genotype & Patient number \\
\hline p.R408W homozygous & $\begin{array}{c}3,9,11-13,16,17,19,25,26,30-33,35,38,39 \\
42-46,55,59,61,63,65-67\end{array}$ \\
\hline p.R408W/c.1066-11G >A & $2,4,6,20,22,34,57,58$ \\
\hline p.R408W/not identified & $24,40,60,64$ \\
\hline p.R408W/c.1315+1G >A & $21,28,53$ \\
\hline p.R408W/c.822-832delGCC & 7,8 \\
\hline \multicolumn{2}{|l|}{ CATGTATA } \\
\hline p.R408W/c.441+5G > T & 10,50 \\
\hline p.R408W/p.P281 L & 48,54 \\
\hline p.R408W/c.47-48deICT & 5 \\
\hline p.R408W/c.1066-3C > T & 14 \\
\hline p.R408W/c. $842+1 G>A$ & 18 \\
\hline p.R408W/c.1269delC & 27 \\
\hline p.R408W/c.353-1G>A & 29 \\
\hline p.R408W/p.R111X & 36 \\
\hline p.R408W/p.Y386C & 37 \\
\hline p.R408W/p.R252W & 41 \\
\hline p.R408W/p.Y414C & 51 \\
\hline p.R408W/p.E390G & 1 \\
\hline c.822-832delGCCCATGTATA/ & 15 \\
\hline \multicolumn{2}{|l|}{ p.I306 V } \\
\hline c.822-832delGCCCATGTATA & 47 \\
\hline \multicolumn{2}{|l|}{ homozygous } \\
\hline c. $1315+1 G>A / p . Y 166 X$ & 49 \\
\hline c. $1315+1 G>A / p . P 281 L$ & 23 \\
\hline c. $1315+1 \mathrm{G}>\mathrm{A} /$ not identified & 52,62 \\
\hline p.R243Q homozygous & 56 \\
\hline
\end{tabular}

identify the second mutation in the five patients, where but a single variant was observed in the coding and adjacent intron regions, analysis for deletions and further assessment of the promoter sequences and sequences deeper within introns would be appropriate.

Among the PKU patients from western Poland, 94\% of the mutations were within or adjacent to exons 7, 11 and 12. Overall, the four most common mutations (p.R408W, c.1066-11G > A, c.1315+1G > A, c.822-832delGCCCATGTATA) accounted for $83 \%$ of the alleles. The mutations p.R408W, c.1066-11G $>$ A and c.1315+1G $>$ A are associated with classical disease in homozygous or hemizygous form. The novel c.822-832delGCCCATGTATA mutation shifts the reading frame to create downstream termination codons likely to activate the nonsense-mediated mRNA decay pathway, thus this mutation is assumed to be severe. The only other novel mutation identified was c.4748delCT in exon 1 that shifts the reading frame creating termination codons; therefore this mutation is also assumed to be severe. Among the 14 other variants the following eight are associated with severe outcomes: c.1269delC, c.353-1G>A, c.441+5G > T, c.842+1G >A, p.R111X, p.Y166X, p.R252W and p.P281L. These variants shift the reading frame, disrupt canonical splice donor/acceptor sites, introduce termination codons or produce inactive enzymes. ${ }^{22,23}$ Jaruzelska

Table 3 Response to $\mathrm{BH}_{4}$ challenge in patients 1-19

\begin{tabular}{|c|c|c|c|c|}
\hline Patient & Genotype & $\begin{array}{c}\text { Blood Phe mg } \\
\text { per } 100 \mathrm{ml}\end{array}$ & $\%$ Reduction & Latency \\
\hline 1 & p.R408W/p.E390G' & $11.67 \rightarrow 2.28$ & 81 & 24 \\
\hline 2 & p.R408W/c.1066-11G >A & $33.72 \rightarrow 28.09$ & 17 & 4 \\
\hline 3 & p.R408W/p.R408W & $37.39 \rightarrow 35.80$ & 4 & 24 \\
\hline 4 & p.R408W/c.1066-11G >A & $30.34 \rightarrow 28.21$ & 7 & 4 \\
\hline 5 & p.R408W/c.47-48delCT & $34.19 \rightarrow 31.43$ & 8 & 8 \\
\hline 6 & p.R408W/c.1066-11G >A & $42.81 \rightarrow 33.20$ & 22 & 8 \\
\hline 7 & p.R408W/11bp del & $35.34 \rightarrow 32.80$ & 7 & 4 \\
\hline 8 & p.R408W/11bp del & $41.62 \rightarrow 32.92$ & 21 & 8 \\
\hline 9 & p.R408W/p.R408W & $48.93 \rightarrow 38.57$ & 21 & 24 \\
\hline 10 & p.R408W/c.441+5G > T & $9.31 \rightarrow 13.66^{c}$ & No reduction & 24 \\
\hline 11 & p.R408W/p.R408W & $42.08 \rightarrow 50.30$ & No reduction & 24 \\
\hline 12 & p.R408W/p.R408W & $24.08 \rightarrow 25.42$ & No reduction & 24 \\
\hline 13 & p.R408W/p.R408W & $30.12 \rightarrow 27.46$ & 9 & 4 \\
\hline 14 & p.R408W/c. $1066-3 \mathrm{C}>\mathrm{T}^{+}$ & $18.31 \rightarrow 16.67$ & 9 & 8 \\
\hline 15 & p.I306V +11 bp del & $7.80 \rightarrow 2.10$ & 74 & 8 \\
\hline 16 & p.R408W/p.R408W & $44.59 \rightarrow 40.56$ & 9 & 24 \\
\hline 17 & p.R408W/p.R408W & $24.18 \rightarrow 18.22$ & 25 & 24 \\
\hline 18 & p.R408W/c.842+1G >A & $38.76 \rightarrow 36.80$ & 5 & 4 \\
\hline 19 & p.R408W/p.R408W & $30.36 \rightarrow 29.71$ & 2 & 4 \\
\hline
\end{tabular}

Table 2 Recurrent mutations in PKU patients from Poznan, Poland and adjacent regions

\begin{tabular}{|c|c|c|c|c|c|c|}
\hline Region & p.R408W & c. $1066-11 G>A$ & c. $1315+1 G>A$ & 11 bp deletion ${ }^{\mathrm{a}}$ & Other common variants & Reference \\
\hline Poland ${ }^{b}$ & $68 \%$ & $6.0 \%$ & $5.2 \%$ & $3.7 \%$ & NA & This study \\
\hline Czech Republic & 54.9 & $1.9 \%$ & $4.1 \%$ & - & p.R158Q (3.85\%) & Kozak et al. ${ }^{18}$ \\
\hline Ukraine & $55 \%$ & $1 \%$ & $1 \%$ & - & p.R158Q (4\%) & Zschocle et al. ${ }^{17}$ \\
\hline Slovakia & $46 \%$ & NR & $10 \%$ & - & p.R261Q (7\%) & Zschocle et al. ${ }^{17}$ \\
\hline Lithuania & $73.4 \%$ & $0.5 \%$ & NR & - & p.R158Q (7.1\%) & Kasnauskiene et al. ${ }^{15}$ \\
\hline Eastern Germany & $38.1 \%$ & $5.7 \%$ & $7.6 \%$ & - & p.R261Q (4.0\%) & Hennermann et al. ${ }^{16}$ \\
\hline
\end{tabular}

Abbreviations: NR, not reported, NA, not applicable

a 11 bp del, c.822-832delGCCCATGTATA.

bPoznan in western Poland. 
et al. ${ }^{24,25}$ assessed PAH genotypes in PKU patients from Poland in 1991 and 1993. Neither study performed a comprehensive analysis of the $P A H$ gene; however, the frequency at which the p.R408W mutation was observed is similar to the frequency observed in this study.

The high frequency of mutations recognized as not responsive to co-factor therapy could be a poor prognosticator to management of PKU patients from western Poland with co-factor therapy. Five of the 67 patients contain variants recognized as responsive to $\mathrm{BH}_{4}$ : p.E390G (patient no. 1), p.R243Q (patient no. 56), c.1066-3C > T (patient no. 14), p.I306V (patient no. 15) and p.Y414C (patient no. 51). ${ }^{5,6,13,14,29}$ Of the 19 patients subjected to the challenge with $\mathrm{BH}_{4}$, patient nos. 1 and 15 (see Table 3) showed significant reduction in their phenylalanine concentration during a single $\mathrm{BH}_{4}$ dose, 24-h assessment. Patient nos. 1 and 15 contain missense mutations that retain residual enzyme activity and are recognized as $\mathrm{BH}_{4}$ responsive alleles. ${ }^{5,14} \mathrm{Had} P A H$ genotype analysis been performed before the $\mathrm{BH}_{4}$ challenge, patient nos. 1 and 15 would have been predicted as likely to respond, whereas genotypes would have predicted 16 of the 17 other patients as non-responsive. Patient no. 14 is compound heterozygous for p.R408W (non-responsive) and the responsive c.1066-3C > T mutation. ${ }^{14}$ Although the c.1066-3C $>\mathrm{T}$ mutation is described as a $\mathrm{BH}_{4}$ responsive, yet there was no reduction of the patients' phenylalanine concentration during the $24 \mathrm{~h} \mathrm{BH}_{4}$ challenge. The c.1066$3 \mathrm{C}>\mathrm{T}$ mutation disrupts the $3^{\prime}$ splice site outside the canonical AG motif (positions -1 and -2 ). The online software resource ESE Finder 3.0 (http://rulai.cshl.edu/cgi-bin/tools/ESE3/esefinder.cgi? process $=$ home) identifies two, high-scoring, exon splice enhancer motifs (GTCACGG, c.1135-c.1141 and CGGAGTT, c.1139-c.1145) for the SR2/ASF serine/arginine-rich splice factor in $P A H$ exon 11 downstream of the mutation. It is possible that these splice enhancers recruit mRNA processing machinery, which may partially alleviate the defect caused by c.1066-3C $>$ T. If the c.1066-3C $>$ T mutation does not fully inactivate the splice site, a reduced level of wild-type $P A H$ mRNA will be produced leading to some wild-type PAH protein. Substrate-activated wild-type $\mathrm{PAH}$ enzyme is further stimulated by $\mathrm{BH}_{4} \cdot{ }^{26}$ The requirement for a $\mathrm{BH}_{4}$ regimen greater than $24 \mathrm{~h}$ has been shown and such may be the case to generate reduced serum phenylalanine with mutations involving splice sites. ${ }^{27,28}$ Repeating the $\mathrm{BH}_{4}$ challenge on patient no. 14 for a longer term $\left(20 \mathrm{mg} \mathrm{kg}^{-1} \mathrm{day}^{-1}\right.$ for $48-120 \mathrm{~h}$ ) may elicit a reduction in phenylalanine concentration not observed in the $24 \mathrm{~h}$ challenge.

Assessment of the $P A H$ gene is a useful diagnostic tool and the genotype has the utility to predict if a patient is a candidate for co-factor therapy. ${ }^{13}$ To summarize, the analysis of the $P A H$ gene in PKU patients from the Poznan region of western Poland, identified a limited spectrum of mutations in which a large majority are both severe and non-responsive to co-factor therapy. The general severity of the mutations may make genotyping prudent before performing the physiological $\mathrm{BH}_{4}$ challenge, as the mutations commonly encountered are generally not associated with $\mathrm{BH}_{4}$-responsive disease.

\section{ACKNOWLEDGEMENTS}

This work was supported by Grant HD075156 to SFD.

1 Guthrie, R. \& Sussi, A. A simple phenylalanine method for detecting phenylketonuria in large populations of newborn infants. Pediatrics 32, 318-322 (1963).

2 Chace, D. H., Kalas, T. A. \& Naylor, E. W. Use of tandem mass spectrometry for multianalyte screening of dried blood specimens from newborns. Clin. Chem. 49, 1797-1817 (2003).
3 Levy, H. L. Phenylketonuria: old disease, new approach to treatment. Proc. Natl Acad. Sci. USA 96, 1811-1813 (1999).

4 Kure, S., Hou, D. C., Ohura, T., Iwamoto, H., Suzuki, S., Sugiyama, N. et al. Tetrahydrobiopterin-responsive phenylalanine hydroxylase deficiency: a novel clinical entity. J. Pediatr. 135, 375-378 (1999).

5 Blau, N. \& Erlandsen, H. The metabolic and molecular bases of tetrahydrobiopterinresponsive phenylalanine hydroxylase deficiency. Mol. Genet. Metab. 82, 101-111 (2004).

6 Levy, H. L., Milanowski, A., Chakrapani, A., Cleary, M., Lee, P., Trefz, FK. \& for the Sapropterin Research Group et al. Efficacy of sapropterin dihydrochloride (tetrahydrobiopterin, 6R-BH4) for reduction of phenylalanine concentration in patients with phenylketonuria: a phase III randomized placebo-controlled study. The Lancet. 370, 504-510 (2007).

7 Muntau, AC., Roschinger, W., Habich, M., Demmelmair, H., Hoffmann, B., Sommerhoff, CP. et al. Tetrahydrobiopterin as an alternative treatment for mild phenylketonuria. N. Engl. J. Med. 347, 2122-2132 (2002).

8 Koch, R., Burton, B., Hoganson, G., Peterson, R., Rhead, W., Rouse, B. et al. Phenylketonuria in adulthood: a collaborative study. J. Inherit. Metab. Dis. 25, 333-346 (2002).

9 Kayaalp, E., Treacy, E., Waters, PJ., Byck, S., Nowacki, P. \& Scriver, CR. Human phenylalanine hydroxylase mutations and hyperphenylalaninemia phenotypes: a meta-analysis of genotype-phenotype correlations. Am. J. Hum. Genet. 61, 1309-1317 (1997).

10 Guttler, F. \& Guldberg, P. Mutation analysis anticipates dietary requirements in phenylketonuria. Eur. J. Pediatr. 159(Suppl 22), S150-S153 (2000).

11 Guldberg, P., Rey, F., Zschocke, J., Romano, V., Francois, B., Michiels, L. et al. A European multicenter study of phenylalanine hydroxylase deficiency: classification of 105 mutations and a general system for genotype-based prediction of metabolic phenotype. Am. J. Hum. Genet. 63, 71-77 (1998).

12 Dobrowolski, SF., Ellingson, CE., Coyne, T., Grey, J., Martin, R., Naylor, E. W. et al. Mutations in the phenylalanine hydroxylase gene identified in 95 patients with phenylketonuria using a novel system of mutation scanning and specific genotyping based thermal melt profiles. Mol. Gen. Metab. 91, 218-227 (2007).

13 Trefz, F. K., Scheible, D., Götz, H. \& Frauendienst-Egger, G. Significance of genotype in tetrahydrobiopterin-responsive phenylketonuria. J. Inherit. Metab. Dis. 32, 22-26 (2009).

14 Zurflüh, M. R., Zschocke, J., Lindner, M., Feillet, F., Chery, C., Burlina, A. et al. Molecular genetics of tetrahydrobiopterin-responsive phenylalanine hydroxylase deficiency. Hum. Mutat. 29, 167-175 (2008).

15 Kasnauskiene, J., Giannattasio, S., Lattanzio, S., Cimbalistiene, L. \& Kucinskas, V. The molecular basis of phenylketonuria in Lithuania. Hum. Mutat. 21, 398 (2003) mutations in brief no. 584 .

16 Hennermann, J. B., Vetter, B., Wolf, C., Windt, E., Buhrdel, P., Seidel, J. et al. Phenylketonuria and hyperphenylalanemia in eastern Germany: a characteristic molecular profile and 15 novel mutations. Hum. Mutat. 15, 254-260 (2000).

17 Zschocke, J. Phenylketonuria mutations in Europe. Hum. Mutat. 21, 345-356 (2003).

18 Kozak, L., Blazkova, M., Kuhrova, V., Pijackova, A., Ruzickova, S. \& St'astna, S. Mutations and haplotype analysis of phenylalanine hydroxylase alleles in classical PKU patients from the Czech Republic: identification of four novel mutations. J. Mol. Genet. 34, 893-898 (1997).

19 Heath, EM., O'Brien, DP., Banas, R., Naylor, E. W. \& Dobrowolski, S. Optimization of an automated DNA purification protocol for neonatal screening. Arch. Pathol. Lab. Med. 123, 154-1160 (1999).

20 Dobrowolski, S. F., Ellingson, C., Caldovic, L. \& Tuchman, M. Streamlined assessment of gene variants by high resolution melt profiling utilizing the ornithine transcarbamylase gene as a model system. Hum. Mutat. 28, 1133-1140 (2007).

21 Bik-Multanowski, M. \& Pietrzyk, J. J. Single exon deletions in the PAH gene in Polish PKU-patients. Mol. Genet. Metab. 94, 267 (2008).

22 Pey, A. L., Desviat, L. R., Gamez, A. \& Ugarte, M. Phenylketonuria: genotype:phenotype correlation based on expression analysis of structural and functional analysis mutations in PAH. Hum. Mutat. 21, 370-378 (2003).

23 Gjetting, J., Petersen, M., Guldberg, P. \& Guttler, F. In vitro expression of 34 naturally occurring mutant variants of phenylalanine hydroxylase: correlation with metabolic phenotypes and susceptibility toward protein aggregates. Mol. Gen. Metab. 72, 132-143 (2001).

24 Jaruzelska, J., Henriksen, K. F., Guttler, F., Riess, O., Borski, K., Blin, N. et al. The codon 408 mutation associated with haplotype 2 is predominant in Polish families with phenylketonuria. Hum. Genet. 86, 247-250 (1991).

25 Jaruzelska, J., Matuszak, R., Lyonnet, S., Rey, F., Rey, J., Filipowicz, J. et al. Genetic background of clinical homogeneity of phenylketonuria in Poland. J. Med. Genet. 30, 232-234 (1993).

26 Kure, S., Sato, K., Fujii, K., Aoki, Y., Suzuki, Y., Kato, S. et al. Wild-type phenylalanine hydroxylase activity is enhanced by tetrahydrobiopterin supplementation in vivo: an implication for therapeutic basis of tetrahydrobiopterin-responsive phenylalanine hydroxylase deficiency. Mol. Genet. Metab. 83, 150-156 (2004).

27 Fiege, B., Bonafe, L., Ballhausen, D., Baumgartner, M., Thony, B., Meili, D. et al. Extended tetrahydrobiopterin loading test in the diagnosis of cofactor-responsive phenylketonuria: a pilot study. Mol. Genet. Metab. 86(Suppl 1), S91-S95 (2005).

28 Shintaku, H., Kure, S., Ohura, T., Okano, Y., Ohwada, M., Sugiyama, N. et al. Long-term treatment and diagnosis of tetrahydrobiopterin-responsive hyperphenylalaninemia with a mutant phenylalanine hydroxylase gene. Pediatr Res 55, 425-430 (2004).

29 Leuzzi, V., Carducci, C., Carducci, C., Chiarotti, F., Artiola, C., Giovanniello, T. et al. The spectrum of phenylalanine variations under tetrahydrobiopterin load in subjects affected by phenylalanine hydroxylase deficiency. J. Inherit. Metab. Dis. 29, 38-46 (2006). 